\title{
Efficiency of Health Sector in South Asia - Some Evidences from Stochastic Frontier Analysis
}

\section{Dar $\mathrm{KH}^{*}$}

Department of Economics, Central University of Kashmir, Jammu and Kashmir, India

\begin{abstract}
South Asia is home to 1709.723 million people and is the most densely populated region of the globe. The GDP of South Asia in terms of PPP is $\$ 9876.6$ bn and in the recent past it came to limelight mainly because of high growth rate of GDP (3.3\%) over the last fifteen years. This high growth rate was mainly fuelled by India. Despite the improvements it has made in the past 25 years it is still categorised as medium human development region with its HDI score of 0.599 . Low human development in south Asia is attributed to its low standard of life, low quality of education and health. The health outcomes of South Asia are relatively poor compared with other regions of the world. The IMR of South Asia is 34.1, MMR is 176 and the DALY's are 712,139.8. South Asia has not only relatively poor health outcomes compared with the other regions of the globe level but there are significant variations in the health outcomes of the constituting countries. In this region the health outcomes of Sri Lanka are better and can be compared to any developed country of the world. The better health outcomes of Sri Lanka are explained by its strong social determinants of health and its efficiency to use the health inputs. To improve the health outcomes mainly by India, Pakistan and Bangladesh which constitute $95 \%$ population of South Asia it is imperative to improve the social determinants and efficient use of health inputs.
\end{abstract}

Keywords: Health status; Health outcome; Human development; South Asia; Efficiency

\section{JEL Classification: I11, I15 and J13}

\section{Introduction}

South Asia comprises of eight countries Afghanistan, Bangladesh, Bhutan, India, Maldives, Nepal, Pakistan and Sri Lanka. These countries are also known as SAARC (South Asian Association for Regional Cooperation) countries. South Asia is the most populous region of the globe supporting almost one fourth of the global population (1.749 billion). In terms of land surface area it constitutes only $3.4 \%$ of the global land surface only. Comparatively low land surface area supporting huge population makes it a highly densely populated region of the world. The population density of this region is 350.6. About 24.6 $\%$ of the South Asian population is living below $\$ 1.25$ a day. The gross domestic product (GDP) per capita PPP international \$ is highest in Maldives (12636.5) and Sri Lanka (11738.8). It is lowest in Afghanistan (1934.1) and Nepal (2458.1) [1]. Population living Below Poverty Line (BPL) ranges from $8.9 \%$ in Sri Lanka to $31.5 \%$ in Bangladesh [2]. About $70 \%$ of the South Asian population and about $75 \%$ of South Asia's poor live in rural areas and mostly depend on agriculture for their livelihood.

In terms of Human Development Index (HDI); a comprehensive indicator of development South Asia still falls in the category of medium human development group with its HDI value of 0.599 . There has been a marginal increase in HDI score of South Asia from 0.503 in 2000 to 0.599 in 2014. Three countries i.e. Nepal, Pakistan and Afghanistan fall in the group of low human development countries with HDI scores of $0.548,0.538$ and 0.465 respectively. India, Bhutan and Bangladesh fall in the category of medium human development countries with their HDI score of 0.602, 0.605 and 0.599 respectively. Only Sri Lanka and Maldives have high human development indicators. The HDI scores of these two countries are 0.757 and 0.706 respectively (UNDP, 2015).

South Asia along with the Sub Sahara Africa is the dark spots on the globe when it comes to health outcomes. The mortality and morbidity indicators of South Asia determine the magnitude of these indicators at the global level. The life expectancy at birth in South Asia is 69 compared to 71.4 at world level. The Infant Mortality Rate (IMR) of South Asia (34.1) is equal to global level (34.1). The Maternal Mortality Rate (MMR) of South Asia is (176) lower than global level (216).

By looking at the progress made by South Asian countries from 2000 to 2015 it is evident that there has been an uneven improvement in the health outcomes of these countries. The IMR of South Asia was 60.25 in 2000 it has been reduced to 34.1 in 2015. Similarly MMR has been reduced from 421.2 to 178.5 in the past fifteen years. The average life expectancy has been improved from 62.9 in 2000 to 69 in 2015. The Disability Adjusted Life Years (DALY's) have been reduced from 785508.2 in 2000 to 712139.8 in 2012.

The status and variation in mortality and morbidity indicators of South Asian countries is given in Table 1 .

From Table 1 it is clear that all the South Asian countries have reduced the MMR and IMR in past fifteen years. From 2000 to 2015 the highest reduction in MMR was achieved by Bhutan $65 \%$ and lowest reduction by Pakistan 41.1\%. From 2000 to 2015 highest reduction in IMR was achieved by Maldives $79.1 \%$ and lowest was achieved by Pakistan 25\%. Despite this reduction in MMR, South Asia still contributes $(66,000)$ maternal deaths which is $21 \%$ of the global maternal deaths. In South Asia, India is the major contributor of the maternal deaths (45000); it contributes 69\% maternal deaths in South Asia and $15 \%$ of global maternal deaths. This huge number of maternal deaths could be brought down only if there is an improvement in the

*Corresponding author: Khursheed Hussain Dar, Department of Economics, Central University of Kashmir, Jammu and Kashmir, India, Tel: +917889892632 E-mail: khursheedarreh@gmail.com

Received December 10, 2018; Accepted December 26, 2018; Published December 31, 2018

Citation: Dar KH (2018) Efficiency of Health Sector in South Asia - Some Evidences from Stochastic Frontier Analysis. Health Econ Outcome Res Open Access 4: 159 doi: 10.4172/2471-268x/1000159

Copyright: (c) 2018 Dar KH. This is an open-access article distributed under the terms of the Creative Commons Attribution License, which permits unrestricted use, distribution, and reproduction in any medium, provided the original author and source are credited. 
determinants of MMR so as to bring them to the level of neighbouring country Sri Lanka. Given the strong correlation between IMR and MMR (0.76) it is imperative to bring improvements in the determinants of these two variables. The main determinants of these two variables are: standard of living measured in GDP per capita, nutrition of expecting mothers, immunization rates, female literacy, access to safe drinking water and proper sanitation, availability and use of effective primary health care etc. In South Asia there is a considerable difference in these determinants across different countries. Percentage of population using improved sanitation facilities ranges from $95.1 \%$ in Sri Lanka to 30\% in Afghanistan. The corresponding figure for India is 39\%. Female literacy an important determinant of MMR because of its linkages with other determinants ranges from $91 \%$ in Sri Lanka to 24\% in Afghanistan. For India female literacy is $62 \%$. Similarly births attended by skill birth attendants ranges from $98 \%$ in Sri Lanka to $41 \%$ in Bangladesh. For India skill birth attendants' rate is $74 \%$. If we look at the DALY's share of South Asia in total global DALY's is $25 \%$. There has been a decline of 9\% in DALY's of South Asia from 2000 to 2012. The highest decline in DALY's was achieved by Maldives (26\%) and lowest decline was achieved by Pakistan (1.95\%). India's share in DALY's of South Asia is $75 \%$ and its share in global level is $19 \%$. There has been $8 \%$ reduction in DALY's in India from 2000 to 2012.

\section{Review of Related Literature}

The outcome of any production process is mainly explained by the resource use efficiency of that production unit. The World health report (WHR) 2010 estimated that about $20 \%-40 \%$ of all health sector resources are wasted and highlighted leading sources of inefficiency [3]. To get the resource use efficiency of any production process, benchmarking finds wide applicability. Benchmarking or relative performance evaluation is defined as the systematic comparison of the performance of one production unit to other units. Different researchers have obtained different efficiency scores using a multitude of efficiency measures across different countries of South Asia. On the basis of Stochastic Frontier Analysis (SFA), Japan with efficiency score of 0.994 ranked at the top followed by China with the efficiency score of 0.993 . India with the efficiency score of 0.919 ranked $66^{\text {th }}$ ahead of USA with its efficiency score of 0.914 . The estimated elasticity of HALE with respect to health care spending is 0.06 . The coefficient of effect of education shows that the elasticity of HALE with respect to years of education diminishes as the level of education rises. Because the estimated elasticity is 0.07 at 3 years of education and 0.05 at 5 years, after which it is no longer significant [4]. The changes in pure technical efficiency and technology level in China implied that since 1999, the operation and management skills of China's medical service institutions have improved while as the technology level of medical diagnosis and treatment has dropped. Huge differences were observed between different provinces in terms of TFP growth rates. The fastest increase was experienced by Tanjin, with a growth rate of $16.4 \%$, while in Xinjiang it was only $0.9 \%$ [5]. On the basis of SFA the mean efficiency of the fourteen States during 1986-1995 in India was 0.692 . Kerala with a widely recognised commitment towards the development of its social sector and Maharashtra with the fastest growing per capita real income were the two best performers in terms of efficiency in production of health. The economically poor States of Rajasthan, Uttar Pradesh, Madhya Pradesh, and Odhissa were the worst performing States with their efficiency scores of $0.64,0.40,0.339$ and 0.23 respectively [6]. With regard to the efficiency of the States Kerala and Goa are the best States with their efficiency score of .97 and hence serve as frontier. The efficiency scores of worst performing States are Bihar (0.79), Madhya Pradesh (0.84), J\&K (0.89), Jharkhand (0.87) and Uttar Pradesh (0.84) [7].

\section{Model Specification}

To compare the performance of the South Asian countries in health sector and to calculate their relative efficiency scores the concept of SFA (Stochastic Frontier Analysis) is used. The model to be used in this analysis is

$$
\mathrm{Y}_{\mathrm{i}}=\beta_{0}+\beta_{1} \mathrm{X}_{1}+\beta_{2} \mathrm{X}_{2}+\beta_{3} \mathrm{X}_{3+} \beta_{4} \mathrm{X}_{4}+\ldots .+\mathrm{v}_{\mathrm{i}}+\mathrm{u}_{\mathrm{i}}
$$$$
\mathrm{i}=1 \ldots . . \mathrm{n}
$$

Where $Y$ is the output, X's are independent variables, $\beta$ 's are the respective coefficients, $u_{i}$ is the difference between the health function frontier of a country and the best practice technique and it is either 0 or negative; $v_{\text {. }}$ is the statistical error and other random factors and it is either positive, negative or zero. The above model means that the health function frontier of any country is given by

$$
\mathrm{Y}_{\mathrm{i}}=\beta_{0}+\beta_{1} \mathrm{X}_{1}+\beta_{2} \mathrm{X}_{2}+\beta_{3} \mathrm{X}_{3+} \beta_{4} \mathrm{X}_{4}
$$

Provided it uses best technique $(u=0)$ and there are no statistical errors $(\mathrm{v}=0)$. If a country uses best technique but there are statistical errors then the frontier function of that country is given by

$$
\mathrm{Y}_{\mathrm{i}}=\beta_{0}+\beta_{1} \mathrm{X}_{1}+\beta_{2} \mathrm{X}_{2}+\beta_{3} \mathrm{X}_{3+} \beta_{4} \mathrm{X}_{4}+\ldots .+\mathrm{v}_{\mathrm{i}}
$$

The presence of $\mathrm{v}$ means that the frontier functions vary randomly across different countries. On the other hand with no statistical errors the given country's realised output will be equal to or less than the potential frontier output depending on whether $u$ is 0 or negative respectively. With the assumption of $u$ being non positive is used in this analysis [8]. This approach assumes a truncated normal (half normal) distribution for $\mathrm{u}$, along with normal distribution for $\mathrm{v}$. Given the density function for $\mathrm{u}$ and $\mathrm{v}$, the frontier function mentioned above is estimated by maximum likelihood technique. The advantage of estimating the frontier function with the assumption of density functions for $u$ and $\mathrm{v}$ is that it ensures whether the deviation of economic unit's actual output from its potential output is mainly because it did not use the best practice technique or is due to external random factors. The stochastic frontier model framed above gives the average technical efficiency measures for the sample observations.

\section{Methodology}

In the current analysis the technical efficiency scores of the South Asian countries is calculated using the data of World Health Organisation [9-11]. The results obtained as such are given in Table 2. In this analysis the dependent/output variable is MMR, because all the main mortality indicators like IMR, CMR and MMR are highly correlated. Besides, South Asia is the region with maximum share of MMR in the world hence makes MMR an important dependent variable for any type of efficiency analysis.

Since SFA is based on the positive outputs, so we need to convert MMR (negative output) into positive output. Theoretically, to convert negative output (MMR) into positive output, it needs to be normalised by taking the difference between its maximum value i.e. 100000 and actual value it attains, it is known as survival index. The limitation with this theoretical normalisation is that practically it is impossible to obtain two extreme values of MMR i.e. 0 and 100000. To overcome this limitation, the maximum and minimum value which MMR could attain is substituted by value of MMR in the country with highest and lowest value in a given year. The highest MMR of 2650 in 2000 and 1360 in 2015 was recorded in Sierra lone. The modified version of survival index i.e. output in the present analysis for the base year i.e. 2005 was taken as the difference between $\left(2650-\mathrm{mmr}_{\mathrm{i}} \mathrm{i}=1 \ldots \ldots 8\right)$ and for the most recent year (2015) it was taken as the difference between $\left(1360-\mathrm{mmr}_{\mathrm{i}} \mathrm{i}=\right.$ 
$1 \ldots \ldots 8)$. Here $i=1 \ldots \ldots . .8$ are the 8 South Asian countries taken for the present study. When we maximise this difference we are minimising the MMR, which is our objective function. The main independent variables influencing MMR are health expenditure measured as percentage of GDP spent on health, institutional deliveries captured by percentage of births attended by skilled birth attendants, percentage of population using improved sanitation, percentage of population using safe drinking water and health infrastructure captured by physician density. These variables explain approximately $90 \%$ variation in the dependent variable i.e. MMR. The results obtained are given in Table 2.

\section{Results and Discussion}

The technical efficiency score of Sri Lanka (0.990) is close to 1 and hence is the most efficient country in South Asia in using health inputs. The efficiency scores of India (0.294), Bangladesh (0.304) and Pakistan (0.219) are almost 3 times less than that of Sri Lanka. Very low efficiency scores of these countries supporting $95 \%$ population of the region is a big challenge. There are ample reasons for the relatively poor performance of these countries. The major reasons explaining these differences are explained below:

Immunization plays a predominant role in avoiding the onslaught of the diseases. In South Asia Srilanka has less than five (U5) mortality (10). This low U5 mortality in major part is explained by $99 \%$ coverage of DPT3, HEPB3 and HIB3 immunization. In Bangladesh U5 mortality reduced from 144 in 1990 to 38 in 2015. Bangladesh has achieved an average annual reduction of 5.9\% from 1990 to 2015 only because it emphasised the immunization and achieved more than $90 \%$ coverage in DPT3, HEPB3 and HIB3 immunization. In Pakistan U5 mortality declined from 139 in 1990 to 81 in 2015. Pakistan has the lowest average annual reduction of U5 mortality of $2.1 \%$ from 2000 to 2015 because it has achieved only $70 \%$ coverage in DPT3, HEPB3 and HIB3 immunization. India launched mass campaigning against the eradication of polio and attained the desired results in 2015. But polio

\begin{tabular}{|c|c|c|c|c|c|c|}
\hline \multirow{2}{*}{} & \multicolumn{3}{|c|}{$\mathbf{2 0 0 0}$} & \multicolumn{2}{|c|}{2015} & $\mathbf{2 0 1 2}$ \\
\cline { 2 - 7 } & MMR & IMR & DALY's & MMR & IMR & DALY's \\
\hline Afghanistan & 1100 & 95.4 & 20598.5 & 396 & 66.3 & 19675.4 \\
\hline Bangladesh & 399 & 64.4 & 65824.4 & 176 & 30.7 & 51230.8 \\
\hline Bhutan & 423 & 59 & 343.7 & 148 & 27.2 & 285.4 \\
\hline India & 374 & 66.4 & 591359.4 & 174 & 37.9 & 539230.9 \\
\hline Maldives & 163 & 35.6 & 93.4 & 68 & 7.4 & 69.1 \\
\hline Nepal & 548 & 59.6 & 13408.5 & 258 & 29.4 & 10284.6 \\
\hline Pakistan & 306 & 87.7 & 86929.9 & 178 & 65.8 & 85231.3 \\
\hline Sri Lanka & 57 & 14 & 6950.4 & 30 & 8.4 & 6132.3 \\
\hline \multicolumn{7}{|c|}{ Source: WHO } \\
\hline
\end{tabular}

Table 1: Mortality and morbidity indicators of South Asian countries, 2000-2015.

\begin{tabular}{|c|c|c|c|c|c|}
\hline Country & $\begin{array}{c}\text { Tech. } \\
\text { Efficiency } \\
\mathbf{2 0 0 0}\end{array}$ & Rank & $\begin{array}{c}\text { Tech. } \\
\text { Efficiency } \\
\mathbf{2 0 1 5}\end{array}$ & Rank & $\begin{array}{c}\text { Change in } \\
\text { ranking }\end{array}$ \\
\hline Afghanistan & 0.25647802 & 6 & 0.22944566 & 6 & 0 \\
\hline Bangladesh & 0.82230322 & 4 & 0.30479785 & 4 & 0 \\
\hline Bhutan & 0.51717144 & 5 & 0.37933414 & 3 & +2 \\
\hline India & 0.13916154 & 8 & 0.29456425 & 5 & +3 \\
\hline Maldives & 0.97061060 & 1 & 0.98645031 & 2 & -1 \\
\hline Nepal & 0.20131650 & 7 & 0.21376433 & 8 & -1 \\
\hline Pakistan & 0.85417050 & 3 & 0.21908854 & 7 & -4 \\
\hline Srilanka & 0.93552389 & 2 & 0.99041797 & 1 & +1 \\
\hline $\begin{array}{c}\text { Mean } \\
\text { efficiency }\end{array}$ & 0.39848867 & & 0.45223288 & & \\
\hline
\end{tabular}

Table 2: Technical efficiency scores of South Asian countries in 2000 and 2015. has not been eradicated completely from Pakistan and Afghanistan. In these two countries $30 \%$ children do not get vaccination of Polio. In rest countries of South Asia this percentage is less than 8 .

There are marked differences across the South Asian countries when it comes to nutrition. The prevalence of anaemic women in the age group 15-49 ranges from 25\% in Srilanka to 51\% in Pakistan. The percentage of anaemic women in the same age group in India is 48 and in Bangladesh it is $43 \%$. India, Pakistan and Bangladesh are home to 203.48 million under nourished people. In India the number of undernourished people is 194 million making it the country with highest number of hungry people in world. Similarly Pakistan's tragedy is that the undernourished population has increased from 28.7 million in 1990 to 41.3 million in 2015.

Access to healthcare services determines the health outcomes of any country. The percentage of women who receive 4 antenatal checkups in Srilanka is 94 . Almost $72 \%$ expecting mothers in India, $25 \%$ in Bangladesh and 37\% in Pakistan receive 4 antenatal check-ups. In Srilanka the skilled birth attendant rate is $99 \%$. The corresponding figures for India, Bangladesh and Pakistan are $67 \%, 44 \%$ and $52 \%$ respectively. This near about $50 \%$ of births not attended by the skilled birth attendants is responsible for high maternal and neonatal mortality in these countries. Proper sanitation is an important determinant of maternal and child health outcomes. In Srilanka population having access to improved sanitation is $94 \%$. In Pakistan it is $48 \%$, Bangladesh it is $57 \%$ and in India it is $36 \%$. Open defecation which generates many health hazards is common in India.

The public health expenditure on health in India, Pakistan and Bangladesh is below $30 \%$ which is very low compared to other countries of the region. This low public health expenditure is another important factor in explaining the differences across these countries given the fact that these three constitute 432.68 million people living below poverty line. For these 432 million people spending $60 \%$ of the expenditure from their pockets is not going to make health their priority.

Education which has huge influence on the health outcomes of any country is also very poor in these three countries. Female literacy which influences particularly maternal and child health outcomes is very low in these countries. Female literacy in India is $62.8 \%$, Bangladesh is $58.5 \%$, and in Pakistan it is $42.7 \%$.

\section{Policy Suggestion}

To sum up it is concluded that in order to improve the health outcomes in South Asia the multi-pronged strategy is needed. Focus should be on improving the investment in health sector so as to make it a priority sector. Since the mean technical efficiency score in South Asia is 0.45 it needs to be improved by increasing the resource use efficiency.

\section{Contribution of the Study}

Given the fact that South Asia is one of the Grey areas when it comes to health outcomes, this study is an attempt to find the reasons for the poor health outcomes and how these could be improved so as to at least bring them to the level attained by some of the South Asian countries like Sri Lanka etc.

\section{References}

1. http://www.who.int/gho/countries/afg/country_profiles/en/

2. https://www.cia.gov/library/publications/the-world-factbook/

3. http://www.who.int/health_financing/documents/health-system-efficiency/en/ 
Citation: Dar KH (2018) Efficiency of Health Sector in South Asia - Some Evidences from Stochastic Frontier Analysis. Health Econ Outcome Res Open Access 4: 159. doi: 10.4172/2471-268x/1000159

Page 4 of 4

4. Ogloblin C (2011) Health care efficiency across countries: a stochastic frontier analysis. Appl Econ Int Dev 11: 5-14.

5. Wu S, Wang C, Zhang G (2015) Has China's new health care reform improved efficiency at the provincial level? Evidence from a panel data of 31 Chinese provinces. J Asian Pub Pol 8: 36-55.

6. Chakrabarti A, Rao DN (2005) Variation in health expenditures: Is it just income or other factors? Empirical investigation using a panel of Indian States. J Quan Econ 3: 158-179.

7. Dar KH, Bhat TA (2018) Health sector efficiency across Indian states using stochastic frontier analysis. Asian Dev Pol Rev 6: 15-19.

8. Aigner D, Lovell CK, Schmidt P (1977) Formulation and estimation of stochastic frontier production function models. J Econom 6: 21-37.

9. https://www.who.int/whr/2000/en/

10. http//hdr.undp.org/en/media/HDR_2015_EN_complete.pdf

11. http//hdr.undp.org/en/media/HDR_2014_EN_complete.pdf 